\title{
A Performance Comparison Study of End-to-End Congestion Control Protocols over MIMO Fading Channels
}

\author{
Homayoun Yousefi'zadeh \\ Department of EECS \\ University of California, Irvine \\ hyousefieuci.edu
}

\author{
Amir Habibi \\ Department of EECS \\ University of California, Irvine \\ ahabibi1@uci.edu
}

\author{
Wojtek Furmanski \\ Integrated Defense Systems \\ The Boeing Company \\ wojtek. furmanski@boeing.com
}

\begin{abstract}
We study the performance of end-to-end congestion control protocols, namely, VCP and TCP/AQM+ECN over wireless topologies created by MIMO fading channel links. We model the loss characteristic of such links with finite-state Markov chains the parameters of which can be derived from the fading channel specifications. We also investigate the effects of utilizing Forward Error Correction (FEC) techniques at the link layer in order to improve the performance of the above congestion control protocols. Our numerical results are generated utilizing NS2 discrete-event simulator. Our results show that without using link layer FEC techniques, the performance of both VCP and TCP/AQM+ECN in wireless networks can be significantly degraded. Further, they show that the use of MIMO links can significantly improve the performance of the protocols of our study.
\end{abstract}

Index Terms-Ad-Hoc Networks, Fading Channel, Markov chain, VCP, TCP, AQM, Performance Evaluation.

\section{INTRODUCTION}

Providing effective packet loss schemes under heavy network loads while adhering to a fair bandwidth allocation notion has been and remains to be a challenging problem. The problem is exaggerated in high bandwidth-delay product environments. The existing end-to-end congestion control scheme built into TCP in conjunction with traditional congestion notification schemes such as Active Queue Management (AQM) and binary Explicit Congestion Notification $(\mathrm{ECN})$ feedback have significant limitations in achieving the above mentioned goal [5], [6], [22], [16], [17]. In order to address the problem, recent literature of congestion control includes, among other proposals, a pair of related end-to-end congestion control protocols. The protocols, namely, eXplicit Congestion-control Protocol (XCP) [18] and Variablestructure Congestion-control Protocol (VCP) [24] can both outperform standard TCP congestion control scheme by isolating fairness from efficiency of congestion control. Both $\mathrm{XCP}$ and VCP achieve high utilization, low persistent queue length, negligible packet loss rate, and reasonable fairness.

This work was sponsored by the grants from Boeing Integrated Defense Systems and UC Discovery Industry-University Cooperative Research Program.
Both protocols require multiple encoded bits of congestionrelated information to be exchanged between routers and end nodes. While the current implementation of XCP [28] requires 16 bits of IP headers, the specification of VCP [24] proposes the use of two ECN bits in the IP header. However, relaying less information in the IP header comes at the cost of a higher response time.

End-to-end signaling in tactical ad-hoc networks networks has to cope with end-to-end encryption challenges. Since packets are encrypted at the point of network entry and decrypted at the point of network departure, very little information can be accessed in a packet while in transit. For example, High Assurance IP Encryption (HAIPE) standard [1] only allows for bypassing six Differentiated Services (DiffServ) bits and two ECN bits across the encryption boundary. Therefore, $\mathrm{VCP}$ and TCP/AQM with binary ECN represent more practical ways of performing end-to-end congestion control than XCP does. In addition, wireless links introduce variable bandwidths and random bit errors in addition to typical packet erasures observed in wired networks. As such, there are three possibilities for a transmitted packet in a wireless environment indicating the packet is (1) delivered error free, (2) lost due to corrupted bits, or (3) lost due to congestion. In [9] and [30], the authors investigate the performance of TCP Reno and Tahoe over wireless links.

This paper investigates the performance of $\mathrm{VCP}$ and $\mathrm{TCP} / \mathrm{AQM}+\mathrm{ECN}$ in wireless networks by comparing them to one another. Our work differentiates from the previous work of [9] and [30] in the sense that (1) it provides a more detailed analysis related to Markov channel modeling of the wireless channel as it pertains to the number of antennas, modulation, channel coding; and (2) it investigates the performance of end-to-end congestion control protocols, namely, VCP and TCP combined with AQM+ECN.

The rest of this paper is organized as follows. Section II describes the characteristics of the physical and data link layers. It explains how the wireless fading channel can be modeled by a finite-state Markov chain the parameters of which are related to physical layer parameters such as the number of transmit/receive antennas and modulation. The section 
also explains how the use of Forward Error Correction (FEC) schemes at the link layer can combat the fading effects. Section III provides a brief description of the transport protocols of interest to this study namely VCP and TCP/AQM+ECN. Section IV contains the results of our experiments and the related discussion. Finally, Section V concludes the paper.

\section{An AnAlysis of PhysicAl AND DATA Link LAYERS}

This section includes a discussion of fading channel modeling with finite-state Markov chains, calculating the symbol error rates of MIMO links, and the channel coding scheme proposed for compensating the effects of correlated loss observed over MIMO links.

\section{A. Finite-State Markov Chain Modeling of MIMO Fading Channels}

As pointed out in our earlier works of [29] and [26] as well as many other articles, a MIMO fading channel is characterized by temporally correlated loss. The two-state GilbertElliott (GE) loss model [10] provides an elegant mathematical model to capture the loss behavior of such channel. In the GE model, the loss behavior of a collection of Units of Information (UoI's) ${ }^{1}$ transmitted over a MIMO fading channel is described by a two-state Markov chain. The GOOD state introduces a probability $\gamma$ of staying in the GOOD state and a probability $1-\gamma$ of transitioning to the BAD state while the BAD state introduces a probability $\beta$ of staying in the BAD state and a probability $1-\beta$ of transitioning to the GOOD state. The parameters $\gamma$ and $\beta$ can be measured from the observed average burst lengths of the wireless channel [15].

The GOOD state also represents the loss of a UoI with probability $\varepsilon_{G}$ while the BAD state represents the loss of a UoI with probability $\varepsilon_{B}$ where $\varepsilon_{B}>>\varepsilon_{G}$. Typical examples of $\varepsilon_{G}$ and $\varepsilon_{B}$ are the average bit, symbol, or packet error rates. For such examples, the pair of probabilities $\varepsilon_{G}$ and $\varepsilon_{B}$ can be calculated from the statistics of the fading channel, transmission powers, average noise power, shadowing, modulation, number of transmit/receive antennas, and even in the presence of co-channel interference. As such, $\varepsilon_{G}$ and $\varepsilon_{B}$ can also be related to quantities such as signal-to-noise ratio or signal-to-interference-noise ratio.

Let $\varphi(t, r, G)$ and $\varphi(t, r, B)$ denote the probability of receiving $r$ Uol's from $t$ transmitted UoI's and winding up in the GOOD and the BAD state of the Gilbert-Elliott model, respectively. Then the overall probability of receiving $r$ UoI's from $t$ transmitted UoI's for the Gilbert-Elliott model is given by

$$
\varphi(t, r)=\varphi(t, r, G)+\varphi(t, r, B)
$$

\footnotetext{
${ }^{1} \mathrm{~A}$ UoI can be thought of as a bit, symbol, or packet.
}

where the recursive probabilities $\varphi(t, r, G)$ and $\varphi(t, r, B)$ are given [29] by

$$
\begin{aligned}
& \varphi(t, r, G)= \\
& \varepsilon_{G}[\gamma \varphi(t-1, r, G)+(1-\beta) \varphi(t-1, r, B)] \\
& \left(1-\varepsilon_{G}\right)[\gamma \varphi(t-1, r-1, G) \\
& \quad+(1-\beta) \varphi(t-1, r-1, B)]
\end{aligned}
$$

and

$$
\begin{aligned}
& \varphi(t, r, B)= \\
& \varepsilon_{B}[(1-\gamma) \varphi(t-1, r, G)+\beta \varphi(t-1, r, B)] \\
& \left(1-\varepsilon_{B}\right)[(1-\gamma) \varphi(t-1, r-1, G) \\
& \quad+\beta \varphi(t-1, r-1, B)]
\end{aligned}
$$

for $t \geq r>0$ and the initial conditions

$$
\begin{aligned}
\varphi(0,0, G) & =g_{s s}=\frac{1-\beta}{2-\gamma-\beta} \\
\varphi(0,0, B) & =b_{s s}=\frac{1-\gamma}{2-\gamma-\beta} \\
\varphi(1,0, G) & =\varepsilon_{G}\left[\gamma g_{s s}+(1-\beta) b_{s s}\right] \\
\varphi(1,0, B) & =\varepsilon_{B}\left[(1-\gamma) g_{s s}+\beta b_{s s}\right]
\end{aligned}
$$

\section{B. Calculation of Symbol Error Rates for MIMO Links}

We now discuss how to calculate the quantities $\varepsilon_{G}$ and $\varepsilon_{B}$ in terms of modulation, number of transmit/receive antennas, and average received signal-to-noise ratios. Our works of [29] and [27] provide the details of extracting the average values of signal-to-noise and signal-to-interference-noise ratios from the statistics of the fading channel, transmission powers, average noise power, shadowing, and in the presence of co-channel interference. While the work of [29] focuses on rich scattering links characterized by Rayleigh fading, the work of [27] captures the dynamics of line of sight links characterized by Rician fading.

In our work, we select the UoI to be a bit in order to provide an accurate physical representation of the wireless MIMO channel. Our work of [26] calculates closed-form expressions describing the modulation symbol error rate of a MIMO wireless link in terms of the number of signal points in the constellation $M$ and the average received signal-tonoise ratio. We carry out our calculations under the assumption of facing a flat fading Rayleigh channel and utilizing PSK modulation. We also note that our approach can be applied to other fading channels such as a Nakagami channel or other modulation schemes such as QAM. In what follows we use our previous results to capture the modulation symbol error rates of a MIMO wireless link.

First, we introduce the modulation symbol error rate of a link associated with single transmit and $N$ receive antenna nodes using Maximum Ratio Combining (MRC) as

$$
\begin{aligned}
& \varepsilon=\frac{M-1}{M}-\frac{1}{\pi} \sqrt{\frac{\vartheta}{1+\vartheta}}\left\{\left(\frac{\pi}{2}+\tan ^{-1} \xi\right) \sum_{j=0}^{N-1}\left(\begin{array}{c}
2 j \\
j
\end{array}\right) \frac{1}{[4(1+\vartheta)]^{j}}\right. \\
& \left.+\sin \left(\tan ^{-1} \xi\right) \sum_{j=1}^{N-1} \sum_{i=1}^{j} \frac{\sigma_{i j}}{(1+\vartheta)^{j}}\left[\cos \left(\tan ^{-1} \xi\right)\right]^{2(j-i)+1}\right\}
\end{aligned}
$$


where $\vartheta=S N R \sin ^{2}\left(\frac{\pi}{M}\right), \xi=\sqrt{\frac{\vartheta}{1+\vartheta}} \cot \frac{\pi}{M}$, and $\sigma_{i j}=$ $\frac{\left(\begin{array}{c}2 j \\ j\end{array}\right)}{\left(\begin{array}{c}2(j-i) \\ j-i\end{array}\right) 4^{i}[2(j-i)+1]}$. From Equation (5), one can calculate the modulation symbol error rate of a link associated with single transmit single receive antenna nodes as well as a link associated with single transmit double receive antenna nodes by setting $N$ to 1 and 2, respectively. Relying on a discussion of diversity gains, we also argue that the modulation symbol error rate of Space-Time Block Codes (STBCs) of [4] and [23] can be calculated from Equation (5) by proper mapping of the values of $S N R$. For example, the modulation symbol error rate of a link associated with double transmit single receive antenna nodes can be calculated by replacing $S N R$ with $\frac{S N R}{2}$ and setting $N$ to 2 in Equation (5). Similarly, the modulation symbol error rate of a link associated with double transmit double receive antenna nodes can be calculated by replacing $S N R$ with $\frac{S N R}{2}$ and setting $N$ to 4 in Equation (5). By selecting $M=2$, Fig. 1 shows BPSK plots of modulation symbol error rate versus average SNR for links with different configurations of transmit and receive antennas. As observed from the plots, the symbol error rates improve going from a single transmit single receive antenna link to a double transmit double receive antenna link. Similar results are observed for other choices of $M=2$.

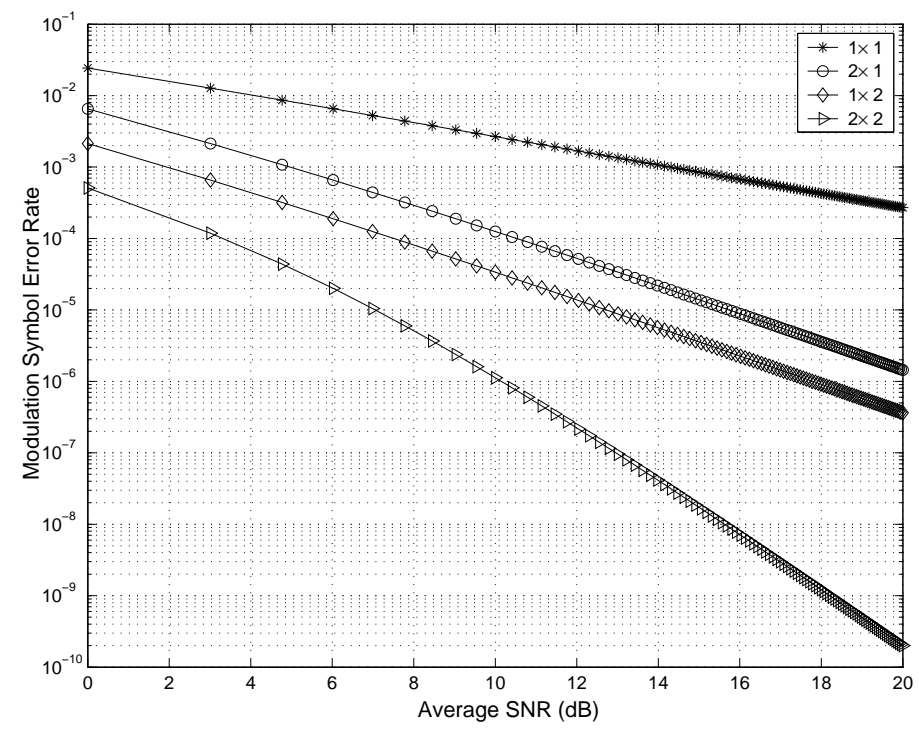

Fig. 1. BPSK plots of modulation symbol error rate versus average SNR for links with different configurations of transmit and receive antennas.

We note that a modulation symbol is mapped to a bit for BPSK. Consequently, it suffices to replace $S N R$ with $S N R_{G}$ and $S N R_{B}$ in Equation (5), in order to calculate modulation symbol error rate $\varepsilon_{G}$ of the GOOD state and $\varepsilon_{B}$ of the BAD state of the GE loss model. We note that while our choices reflect a matter of convenience for our study, they do not affect the generality of our model.

\section{Link Layer Channel Coding}

We open this section by indicating that some standards such as Direct Sequence Multiple Access Code Division (DS-CDMA) [3] have proposed the use of Forward Error Correction schemes at the link layer in order to combat the fading effects described above.

In our work, we propose the use of Reed-Solomon (RS) channel coders at the link layer as an optional component to compensate for temporally correlated loss effects of the wireless channel. We note that an RS channel coder $R S(b, k)$ converts $k$ channel coding symbols into a $b$-symbol block by appending $(b-k)$ parity symbols. Such channel coder is able to correct as many as $t_{C}=\left\lfloor\frac{b-k}{2}\right\rfloor$ symbol errors in a block.

We distinguish between the modulation symbols and channel coding symbols by noting that a channel coding symbol typically consists of a number of modulation symbols. For example, an 8-bit RS channel coding symbol consists of eight BPSK modulation symbols. If at least $b-t_{C}$ channel coding symbols are correctly received from $b$ transmitted channel coding symbols, the whole block is recoverable.

At the end of this section, we note that we are applying an RS channel coder $R S(b, k)$ treating an assembled packet at the data link layer as its data block $k$, Therefore, errors in both the payload and headers/trailers can be mitigated for each packet individually at the receiving side.

\section{An OVERVIEW OF END-TO-END CONGESTION PROTOCOLS}

In this section, we provide a brief overview of the endto-end congestion control protocols of interest to our study, namely TCP+AQM/ECN and VCP. We note that the protocols operate in network and transport layers.

Inspecting the properties of standard TCP reveals that it utilizes Additive-Increase Multiplicative-Decrease (AIMD) property. While the use of AIMD property allows for offering both efficiency and fairness of congestion control, it results in performance degradation of TCP due to the slow start phenomenon. Further and as indicated in many research articles such as the works of [20], [8], TCP does not work well in environments with a high bandwidth-delay products. In order to address some of the limitations of TCP's congestion control scheme, the use of congestion notification feedback schemes such as TCP+AQM/ECN has been proposed [12], [6], [19], [22]. While the use of TCP+AQM/ECN can result in reduced loss rate and queue size, it is still not capable of achieving high utilization in high bandwidth-delay product networks [14], [21], [24].

As such, the state-of-the-art networking research has focused on addressing TCP's congestion control problems. XCP [18] and VCP [24] are a pair of protocols recently proposed to address the problem. Unlike TCP that controls 
both efficiency and fairness of congestion control with one AIMD mechanism, XCP and VCP attempt at decoupling the efficiency of congestion control from its fairness. Such decoupling allows for employing different strategies to cope with efficiency and fairness. The premise of XCP is to use a Multiplicative-Increase Multiplicative-Decrease (MIMD) scheme for efficiency and an AIMD scheme for fairness. The decoupling can hence result in improving the efficiency of congestion control measured in terms of smaller queues; fewer drops; offering flexible fairness schemes such as maxmin or proportional fairness; and providing a better scalability by virtue of not carrying any per-flow state information.

Although XCP represents a great potential in addressing the problems of TCP, it faces some serious challenges. One such challenge of the highest interest to our study is the fact that XCP requires the use of multiple bits in the IP header of each packet. While an implementation of XCP in Linux kernel uses 16 bits [28], the current IETF draft for XCP [11] proposes the use of 128 bits in the IP header. Therefore, the use of XCP in encrypted networks is not practical as current encryption devices only allow for bypassing six DiffServ bits and two ECN bits.

The design goal of Variable-structure Control Protocol (VCP) is to provide a TCP-like scheme for wired networks that requires a small amount of congestion information in the IP header and scales across a wide range of network scenarios. VCP attempts at achieving high efficiency both in low and high bandwidth-delay product environments, low loss, and small queue sizes with a fairness model similar to that of TCP. Using a quantity called network link load factor as the indicator of congestion signal, VCP decouples efficiency and fairness control in different load regions. VCP proposes calculating, quantizing, and encoding the load factor into two ECN bits. It recognizes three operating regions namely, lowload, high-load, and overload. The objective of VCP is to operate in high-load operating region by applying Multiplicative Increase (MI) in low-load region, Additive Increase (AI) in high-load, and Multiplicative Decrease (MD) in overload regions.

As the result of quantization and since the amount of information relayed in the IP header is much smaller compared to $\mathrm{XCP}$, VCP's fairness convergence time is much higher than that of XCP. However, it is much more practical than XCP in terms of implementation.

\section{Performance Evaluation}

In this section, we describe the performance results of the pair of congestion control protocols of our study over MIMO fading networks.

We experiment with a variety of parameter settings. While the trends of reported results are general, the followings rep- resent the various settings of the wireless channel and physical layer for the reported results. The transition probabilities of the GE model are set as $\gamma=0.99875$ and $\beta=0.875$ representing average burst lengths of 800 and 8 bits for the GOOD and BAD state, respectively. Further, we set $S N R_{G}=10 S N R_{B}$ to differentiate between the qualities of the link in the GOOD and BAD states. Four different combinations of antennas are considered as (1) single transmit single receive $(1 \times 1)$; $(2)$ double transmit single receive $(2 \times 1)$; (3) single transmit double receive $(1 \times 2)$; and (4) double transmit double receive $(2 \times 2)$ antennas. We utilize BPSK modulation along with a channel coding symbol size of one byte when using RS coding FEC. We note that the channel coding rate is defined as $r=k / b$ when using FEC.

Fig. 2 illustrates the topology of our experiments. The topology should be thought of as a pair of communication platforms communicating to one another over a MIMO wireless link. Each platform includes a number of wired nodes that are connected to a multiple antenna radio. Each radio acts as the wireless gateway for its platform. While the capacity and the delay of the bottleneck wireless link are assumed to be $10 \mathrm{Mbps}$ and $32 \mathrm{msec}$, the other links are assumed to have a capacity of $20 \mathrm{Mbps}$ and a delay of 6.4 msec. Thus, the communication model resembles a dumbbell topology with the pair of multiple antenna radios forming the bottleneck link.

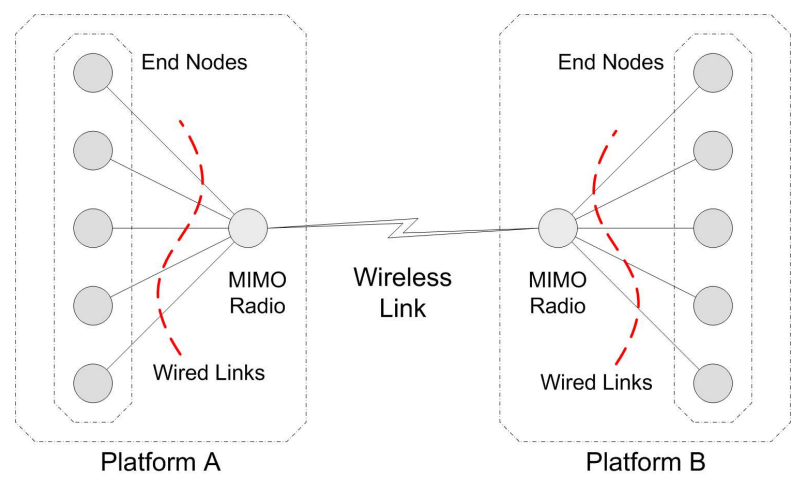

Fig. 2. An illustration of the communication topology used in our experiments.

We set the parameters of $\mathrm{VCP}$ according to what is reported in Table 1 of [24]. In the case of TCP/AQM+ECN, we utilize TCP Reno along with Random Early Marking (REM) [6]. Particularly, we set the parameters of REM according to what is suggested by [7] at the two ends of the bottleneck link.

In all of the following curves, the horizontal axis represents $S N R_{G}$. While the vertical axis represents Packet Error Rate (PER) in Fig. 3 and 4, it shows the total number of packets delivered during the course of simulation in Fig. 5 and 6. All of the simulations are repeated 5 times and averaged with 
individual runs over a total simulation period of 20 seconds.

We select $10 \mathrm{ftp}$ sources in Platform A sending packets to destinations in Platform B and $10 \mathrm{ftp}$ sources in Platform B sending packets to destinations in Platform A. When active, each source generates packets at the rate of its dedicated link. Each data packet has a size of 1040 bytes and each acknowledgment packet has a size of 40 bytes. All of the links operate in a full-duplex mode and link bandwidths are shared among data and acknowledgment packets. Within the 20 seconds of an individual run, each flow starts as soon as its session is established and continues until either its session is torn down due to fading- and/or congestion-related loss or the simulation experiment has come to an end. As such, each flow follows a random start time with a random duration. We collect the statistics in the last 10 seconds of each run to allow for stabilizing the transitioning behavior of NS2.

As our baseline scenario, we consider the performance of VCP over a $1 \times 1$ antenna configuration with no FEC at the link layer. Fig. 3 compares the performance of our baseline case with those of other antenna configurations. As illustrated, all of the curves exhibit saturation-like behavior directly related to the quality of the channel. For small values of $S N R_{G}$ representing a low channel quality, the value of PER is close to $100 \%$. As the quality of channel improves, the value of PER improves eventually approaching zero. When comparing the results of different antenna configurations, we observe that the performance of $1 \times 1,2 \times 1$, $1 \times 2$, and $2 \times 2$ are in an ascending order as the result of improving SER from the former to the latter configuration.

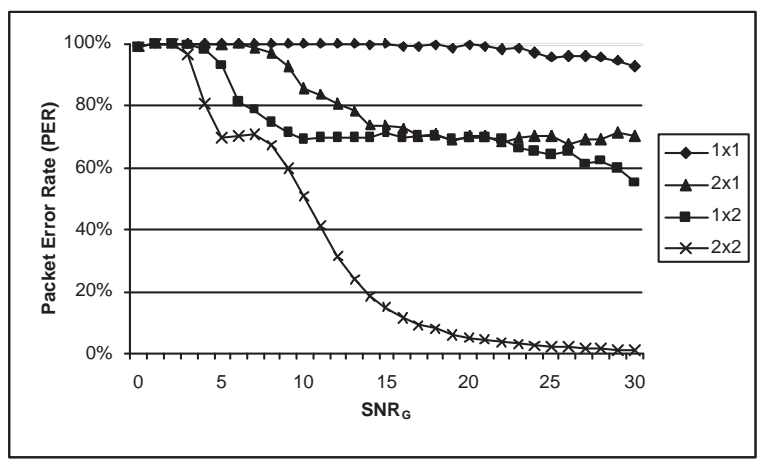

Fig. 3. A comparison of VCP performance with different antenna configurations of the wireless link. No link layer FEC is applied.

Fig. 4 examines the effects of adding link layer FEC for $1 \times 1$ and $2 \times 2$ antenna configurations. While not shown due to the shortage of space, we have observed similar results for other antenna configurations. Comparing different antenna configurations, we see once more that the performance of $1 \times 1,2 \times 1,1 \times 2$, and $2 \times 2$ are in an ascending order. It is observed from the figures that introducing a small percentage of FEC at the link layer can significantly improve the performance of VCP. The latter is due to the fact that VCP sources collect information about the status of the network from the acknowledgment packets. Since acknowledgment packets are only 40 bytes long and fading related random errors happen in bursts, they can corrupt the shorter acknowledgment packets much easier than longer data packets. As important as it is to protect the contents of acknowledgment packets, increasing the strength of the FEC beyond a certain rate may not offer proportional performance improvement due to the bandwidth overhead accrued by data packets. As such, the FEC strength must be chosen with the consideration of antenna configuration and link quality. It is our opinion based on experiments that providing stronger protection for acknowledgment packets as opposed to data packets can properly address the tradeoff between protocol efficiency and bandwidth overhead.

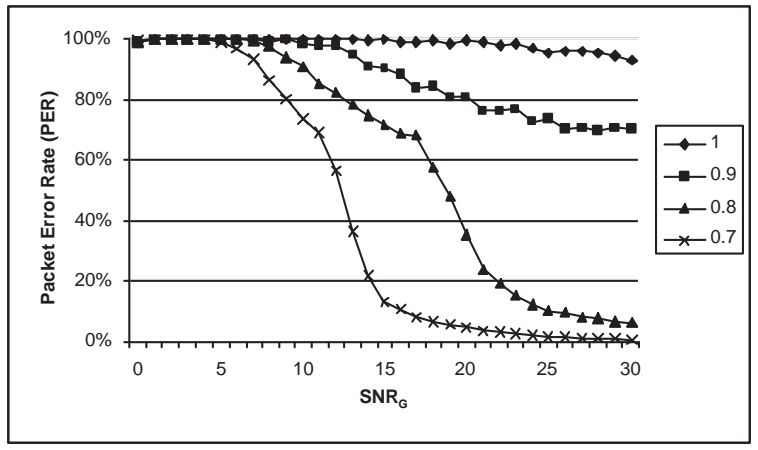

(a)

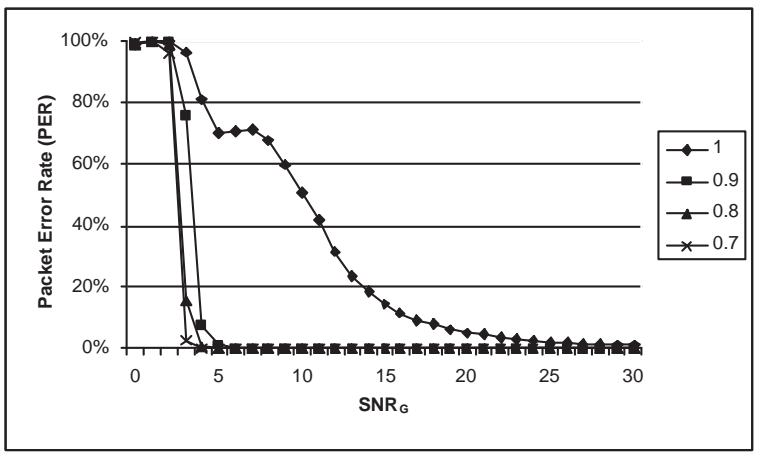

(b)

Fig. 4. A comparison of VCP performance. Reed-Solomon link layer FEC with rates $1,0.9,0.8$, and 0.7 is applied over (a) $1 \times 1$ and (b) $2 \times 2$ antenna configuration of the wireless link.

Next, we compare the performance of VCP with that of TCP/AQM+ECN with no FEC for different combinations of antennas. The performance is measured in terms of total delivered data packets. Fig. 5 illustrates the comparison results for the cases of $2 \times 1$ and $2 \times 2$ antenna configurations. It is interestingly observed from the figure that TCP/AQM+ECN outperforms VCP by a large margin in terms of the number of delivered data packets. Referring to Fig. 3 and noting that the PER of VCP for a $2 \times 2$ antenna configuration and 
large values of $S N R_{G}$ values is close to zero, the observation requires justification. The observation is justified relying on the fact that the existence of fading related random errors and the lack of FEC corrupts many of the original short acknowledgment packets forcing multiple retries and postponing of the VCP session establishment. On the contrary, the built-in retry mechanism of TCP seems to react to the problem with a higher agility resulting in quicker session establishment. As such, the life span of VCP flows is much lower than that of TCP flows and the total number of data packets transmitted by VCP flows is much lower than the total number of data packets transmitted by TCP flows. Consequently, the total of number of delivered data packets in the case of VCP is much lower than that in the case of TCP although the data PERs are low for large values of $S N R_{G}$ after the session establishment.

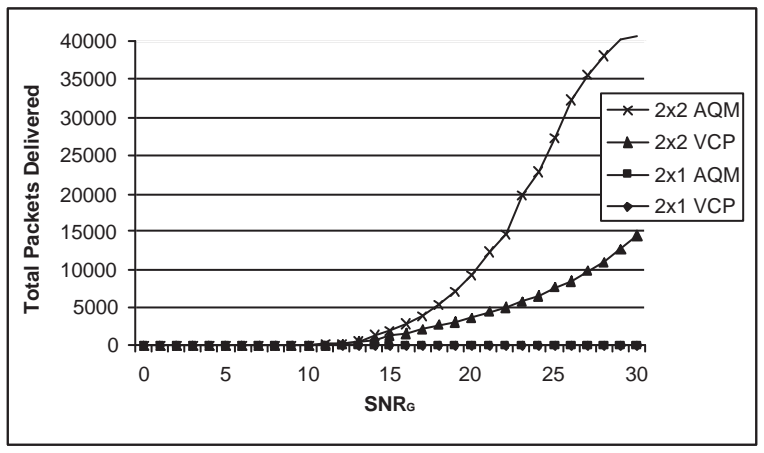

Fig. 5. A performance comparison of $\mathrm{VCP}$ and TCP/AQM+ECN with a $2 \times 1$ and a $2 \times 2$ antenna configuration of the wireless link. No link layer FEC is applied.

Fig. 6 reports the results of similar experiments for FEC rates of $0.9 \%$ and $0.7 \%$. It illustrates the significant effects of applying FEC allowing VCP to outperform TCP/AQM+ECN by a margin of $6 \%$ to $10 \%$. Passed a threshold value for $S N R_{G}$, the curves also show a very robust packet delivery rate for VCP as oppose to somewhat oscillatory packet delivery rate for TCP/AQM+ECN. While not shown, the performance advantage is further augmented as the bandwidthdelay product of the links increases proportional to the flows.

At the end of this section, it is insightful to discuss the effects of a number of parameters. First, we note that the use of larger data packet sizes typically results in requiring to use higher FEC strengths. This is anticipated as larger packet sizes mean a larger collection of channel coding symbols to form a block of data. Second, we note that the use of larger channel coding symbol sizes also results in requiring to use higher FEC strengths due to the fact that a channel burst loss is more likely to corrupt at least one bit of the symbol. However, the above issues are traded off against segmentation/reassembly and the decoding complexity, respectively.

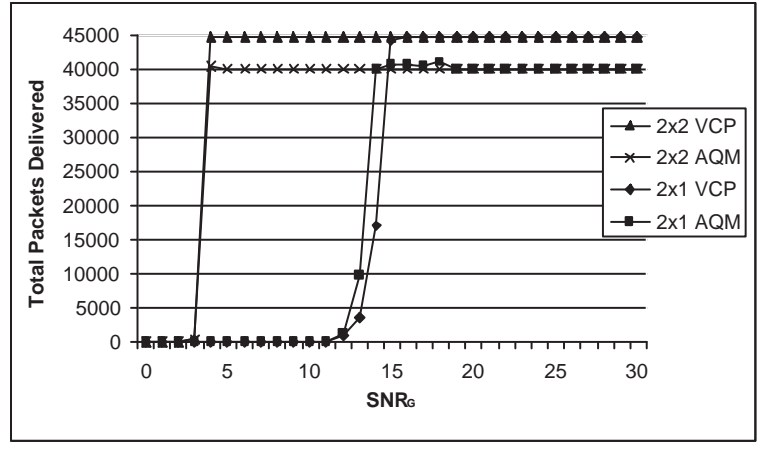

(a)

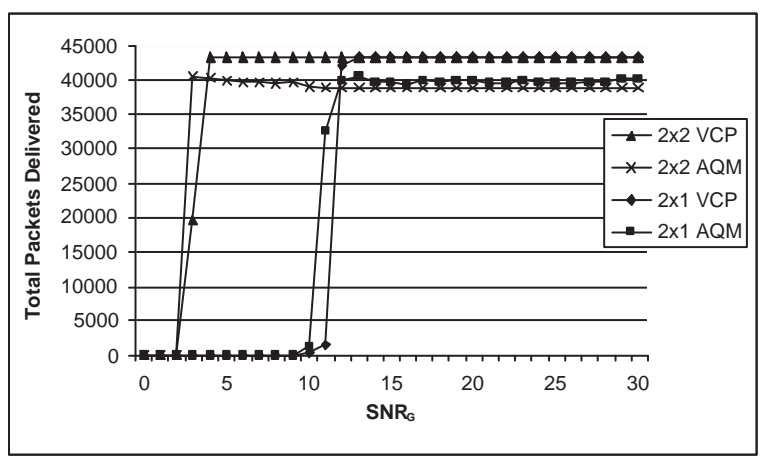

(b)

Fig. 6. A performance comparison of $\mathrm{VCP}$ and TCP/AQM+ECN with a $2 \times 1$ and a $2 \times 2$ antenna configuration of the wireless link. Reed-Solomon link layer FEC with rates (a) 0.93 and (b) 0.7 is applied.

Further, the effects of the larger $\gamma$ and smaller $\beta$ transition probabilities are similar to those of better $S N R_{G}$ and better $S N R_{B}$. However, we have observed that signal-to-noise ratios usually impact PER in a more significant way than the transition probabilities. Finally, we would like to discuss the impacts of having to deal with short-lived flows as oppose to long-lived flows. We have made consistent observations as what is reported by [24] in the case of VCP over wired scenarios, i.e., our PER results have remained the same and the implications have mostly appeared in the form of varying average buffer sizes.

\section{CONCLUSION}

In this paper, we described an experimental study attempting at capturing the performance of VCP and TCP/AQM+ECN over wireless topologies created by fading MIMO channel links. We assumed the existence of random bit errors and packet erasures over such links. We modeled the loss characteristics of such links with finite-state Markov chains the parameters of which could be derived from the fading channel specifications. We also investigated the effects of utilizing FEC techniques at the link layer in order to improve the performance of the above congestion control protocols. Our numerical results showed degradation effects 
of random bit errors in the performance of the congestion control protocols of our study. The results also illustrated that moderate use of link layer FEC techniques could help compensate for such effects. Our results also revealed that VCP outperforms TCP/AQM+ECN in the investigated scenarios of our study given that acknowledgment packets are reasonably protected by link layer FEC.

At the end of this paper, we would like to comment on the stability of VCP protocol. To that end, it is important to mention that the analysis of stability applied to the behavior of VCP in [24] is over simplistic at least in the case of wireless networks. Problems exists as VCP requires periodical measurement of link bandwidths. However, such measurements are subject to estimation errors resulting in oscillatory behavior. Addressing the stability issues of VCP are the subject of our ongoing research work.

\section{REFERENCES}

[1] -, "Global Information Grid Net-Centric Implementation Document: Quality-of-Service (T300)," FOUO PreCoordination Plan Document, August 2005. Available at https://disronline.disa.mil/a/DISR/docs/T300_V1-0_FINAL.pdf.

[2] -, "Network Simulator NS2," Available at http://www.isi.edu/nsnam/ns/.

[3] -, "TIA/EIA/IS-99, Data Services Option Standard for Wideband Spread Spectrum Digital Cellular System," 1995. Available at http://electronics.ihs.com/document/abstract/EZGWCAAAAAAAAAA

[4] S.M. Alamouti, "A Simple Transmitter Diversity Scheme for Wireless Communications," IEEE JSAC, November 1998.

[5] M. Allman, V. Paxson, W. Stevens, "TCP Congestion Control," IETF RFC 2581, April 1999.

[6] S. Athuraliya, V. Li, S. Low, and Q. Yin, "REM: Active Queue Management," IEEE Network, May 2001.

[7] S. Athuraliya, "A Note on Parameter Values of REM with Reno-like Algorithms," Available at . http://netlab.caltech.edu/pub/papers/REMparameter.pdf, March 2002.

[8] S. Bhandarkar, S. Jain, A. Reddy, "Improving TCP Performance in High Bandwidth High RTT Links Using Layered Congestion Control," In Proc. PFLDNet, 2005.

[9] A. Chockalingam, M. Zorzi, R.R. Rao, "Performance of TCP on Wireless Fading Links with Memory," In Proc. IEEE ICC, 1998.

[10] E.O. Elliott, "Estimates on Error Rates for Codes on Burst-Noise Channels,” Bell Syst. Tech. J., September 1963.

[11] A. Falk, D. Katabi, Y. Pryadkin, "Specification for the Explicit Control Protocol (XCP)," IETF Internet Draft, Available at http://www.isi.edu/isi-xcp/docs/draft-falk-xcp-spec-01.txt, October 2005 .

[12] S. Floyd, V. Jacobson, "Random Early Detection Gateways for Congestion Avoidance,” IEEE/ACM Trans. Networking, August 1993.

[13] E.N. Gilbert, "Capacity of A Burst-Noise Channel," Bell Syst. Tech J., September 1960.

[14] C. Hollot, V. Misra, D. Towsley, W. Gong, "Analysis and Design of Controllers for AQM Routers Supporting TCP Flows," IEEE Trans. Automatic Control, June 2002.

[15] H. Jafarkhani, P. Ligdas, N. Farvardin, "Adaptive Rate Allocation in a Joint Source-Channel Coding Framework for Wireless Channels," In Proc. IEEE VTC, 1996.
[16] A. Jain, S. Floyd, “Quick-Start for TCP and IP,” IETF Internet Draft draft-amit-quick-start-02.txt, October 2002.

[17] C. Jin, D. Wei, S. Low, "FAST TCP: Motivation, Architecture, Algorithms, Performance,” In Proc. IEEE INFOCOM, 2004.

[18] D. Katabi, M. Handley, C. Rohrs, "Congestion Control for High Bandwidth-Delay Product Networks," In Proc. of ACM SIGCOMM, 2002.

[19] S. Kunniyur, R. Srikant, "Analysis and Design of an Adaptive Virtual Queue (AVQ) Algorithm for Active Queue Management," In Proc. ACM SIGCOMM, 2001.

[20] T. Lakshman, U. Madhow, "The Performance of TCP/IP for Networks with High Bandwidth-delay Products and Random Loss," IEEE/ACM Trans. Networking, June 1997.

[21] S. Low, F. Paganini, J. Wang, and J. Doyle, "Linear Stability of TCP/RED and a Scalable Control," Computer Networks Journal, December 2003.

[22] K. K. Ramakrishnan, S. Floyd, "The Addition of Explicit Congestion Notication (ECN) to IP," IETF RFC 3168, September 2001.

[23] V. Tarokh, H. Jafarkhani, A.R. Calderbank, "Space-Time Block Coding from Orthogonal Designs," IEEE Trans. Information Theory, July 1999.

[24] Y. Xia, L. Subramanian, I. Stoica, S. Kalyanaraman, "One More Bit Is Enough," In Proc. ACM SIGCOMM, 2005.

[25] L. Ying, G. Dullerud, R. Srikant, "Global Stability of Internet Congestion Controllers with Heterogeneous Delays," In Proc. IEEE ACC, June 2004.

[26] H. Yousefi'zadeh, H. Jafarkhani, M. Moshfeghi, "Power Optimization of Wireless Media Systems with Space-Time Block Codes," IEEE Trans. Image Processing, July 2004.

[27] H. Yousefi'zadeh, "A Constrained Resource Allocation Study for LOS MIMO Fading Ad-Hoc Networks," In Proc. IEEE WCNC, 2006.

[28] Y. Zhang, T.R. Henderson, "An Implementation and Experimental Study of the eXplicit Control Protocol (XCP)," In Proc. IEEE INFOCOM, 2005.

[29] L. Zheng, H. Yousefi'zadeh, H. Jafarkhani, "Resource Allocation in Fading Wireless Ad-Hoc Networks with Temporally Correlated Loss," In Proc. of IEEE WCNC, 2004.

[30] M. Zorzi, A. Chockalingam, R.R. Rao, "Throughput Analysis of TCP on Channels with Memory," IEEE JSAC, July 2000. 